\section{EFFECT OF DIFFERENT DIETARY FATS ON BLOOD COAGULATION, PLATELET ECONOMY, AND BLOOD LIPIDS*}

BY

J. F. MUSTARD, M.D., Ph.D.

Senior Research Associate, National Heart Foundation of Canada

AND

E. A. MURPHY, $\uparrow$ M.D.

From the Department of Medicine, University of Toronto; Sunnybrook Hospital, Department of Veterans Affairs, Toronto; and Department of Physiological Sciences, Ontario Veterinary College, Guelph

The weight of the evidence from many studies indicates that blood-clotting as measured by in vitro tests is accelerated during alimentary lipaemia (O'Brien, 1957 ; Mustard, 1961). O'Brien (1958), however, has emphasized the need for caution in concluding from such results that there are corresponding changes in vivo. Measurement of platelet survival and turnover allows some estimate of in vivo clotting (Murphy and Mustard, 1961). We have therefore studied blood coagulation, platelet survival and turnover, and blood lipids in a group of seven patients maintained successively on each of three rigidly controlled diets for a period of four or more weeks each.

\section{Materials and Methods}

The entire study was conducted in the metabolic unit at Sunnybrook Hospital.

Subjects.-Seven male white Canadian veterans were studied. Their ages ranged between 56 and 65 years. Five of the subjects had suffered from cerebrovascular or cardiovascular complications of atherosclerosis, none of them within five months of the start of the experiment. Of the other two, one had chronic bronchitis and emphysema and the other had quiescent gout. This latter subject received sulphinpyrazone $250 \mathrm{mg}$. b.i.d. throughout the period of the study.

Coagulation Tests.-The whole-blood clotting-time, one-stage prothrombin time, the activity of plasma in place of serum in the thromboplasin generation test, platelet clumping time, platelet count, and platelet adhesive index were carried out by techniques previously described (Mustard, 1956 ; Mustard, 1957 ; Murphy and Mustard, 1960).

\section{Platelet Survival and Turnover}

Platelet survival was determined by the method of Leeksma and Cohen (1956) using modifications (Murphy and Mustard, 1961). This technique is basically the labelling in vivo of platelets with diisopropyl fluorophosphonate containing radioactive phosphorus $\left({ }^{32} \mathrm{P}\right) . \ddagger$

From these results platelet survival was computed first on the assumption that platelets are randomly destroyed

* Supported in part by a U.S. Public Health Service Grant (H-4964).

†Now at the Joseph E. Moore Clinic, Johns Hopkins Hospital, Baltimore, Maryland.

†Obtained from the Radiochemical Centre, Amersham, Buckinghamshire, England. ("exponential pattern"), and secondly the assumption that platelet survival is normally distributed about a mean ("Gaussian or linear pattern"). Recent animal studies have led us to conclude that randomly acting factors are important in determining the life-span of platelets (Robinson, Murphy, and Mustard, 1961, unpublished observations). Platelet turnover in thousands per c.mm. per day was computed on the assumption that platelet production remained steady during the period of each study. The details of the method and calculations have been reported (Murphy and Mustard, 1961, 1962).

It is probably unimportant whether the curve fitted to the plot of the radioactive count against time is a straight line or an exponential, provided the same method is used throughout. We have, in fact, previously shown that there is an excellent correlation between the results obtained by the two methods though there is a gross difference between the two estimates of mean survival so obtained (Murphy and Mustard, 1962). As a more analytical approach to this we have constructed two theoretical curves (Figs. 1 and 2). In the former we have computed the intercept on the $X$ axis of the best linear fit to a series of daily readings expected with a regular exponential decay. It is seen that there is an almost perfect linear relationship between the true half-life and the "linear" estimates of the mean within the limits of the readings which we encounter in platelet survival studies. However, it is of importance that the line obtained depends on the number of days over which readings are taken. Thus for a given exponential halflife, the more readings taken the larger the interceptthat is, the larger the estimate of mean survival. For this reason, in the present and future studies, readings are taken over a period of 10 days.

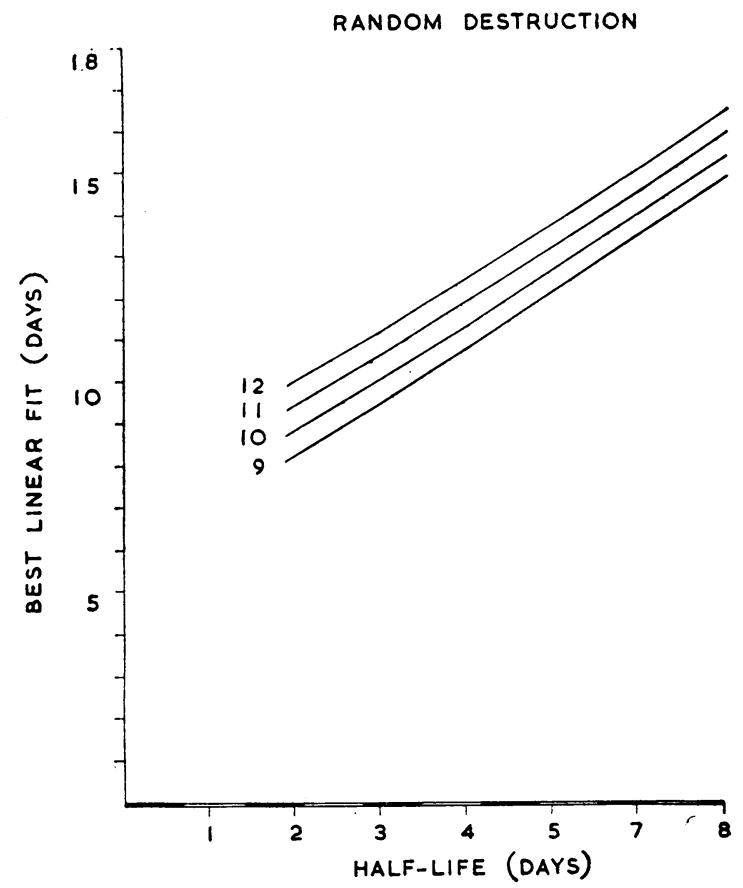

Fig. 1.- On the base line are two scales between which there is an exact correspondence. The figures refer to true half-life in days, and the blocked scale refers to true mean survival computed by dividing the half-life by 0.69315 . On the vertical scale is plotted the estimate of the mean survival based on the erroneous assumption that decay is linear. The curves show the relationship between these depending on whether values for the first $9,10,11$, or 12 days are used. 
In Fig. 2 we have considered the reverse possibilitythat decay is linear, but that survival is computed on the assumption that the decay is exponential. For the latter it has been necessary to exclude all counts equal to zero. This relationship also is in the main linear,
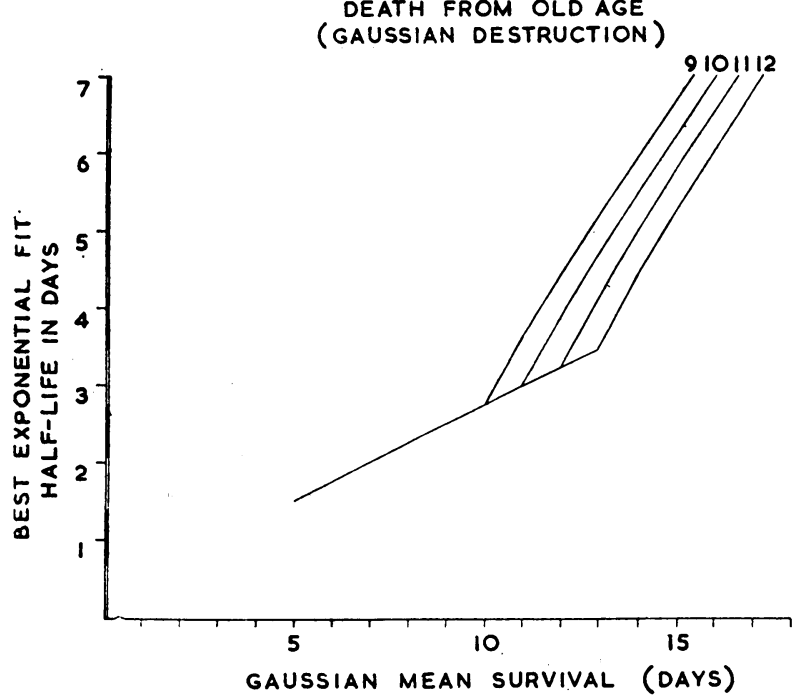

Fig. 2.-On the base line is plotted the true mean survivalthat is, the intercept of a linear decay on the time axis. On the vertical scale are shown estimates of exponential half-life and mean survival (blocked scale) obtained on the erroneous assumption that decay is exponential. For obvious reasons, zero readings have had to be discarded. The main curve shows the relationship between the two quantities. The branching curves relationship between the two quantities. The branching curves first $9,10,11$, or 12 days only. (The true form of the function is in fact much more complicated.)

but it too is sensitive to the number of days over which readings are taken. There is an angularity which arises at the point where there is a change from the use of all the readings except the final zero, to the first $9,10,11$, or 12 daily readings only. The actual departure from linearity is relatively small, but this, too, seems a good reason for using values from 10 days, no more, no less.

Serum Cholesterol and Phospholipid

Serum cholesterol was determined by the technique of Sperry and Webb (1950) and serum phospholipid by the technique of Zilversmit and Davis (1950).

Expression of Results and Statistical Considerations

Mean values for each of the above variables are given in the Tables. We have previously discussed the distribution of the values in the clotting tests and platelet survival and turnover (Murphy and Mustard, 1960 1961, 1962). All are normal except for whole-blood clotting-time and platelet clumping time, which are lognormal, and the thromboplastin generation test, which is harmonic-normally distributed. Appropriate transformations have been made before any statistical calculations were done, but the values given have been transformed back to the original units of measurement before being inserted in the Tables.

It is well known that blood cholesterol has a skewed distribution (Thomas and Eisenberg, 1957), but in the individual the variation is too small for any simple transformation to be of value (Murphy, 1961, unpublished observations). The same was found to be true of phospholipid, about which information available is in general much scantier.

Analysis of the results has been complicated by marked and largely unexplained heterogeneity of variance within the 21 dietary periods which could not be eliminated by transformation. Accordingly comparisons have been made by paired $t$ tests on differences between the means. It has seemed reasonable to treat the comparisons between the three dietary groups as independent, since this corresponds to the nature of the hypotheses set up.

\section{Diets}

Three patients were studied while on each of three diets in the following order: (1) with low fat content, (2) rich in egg yolk and dairy fat, and (3) containing no dairy products and with most of the fats from vegetable sources.

The remaining four patients were studied on the same three diets, but given in the reverse order. Platelet survival studies were done after each subject had been receiving the diet for three or more weeks. Thus they received each diet for a period of more than one month. Samples of blood for lipid analysis and in vitro tests of coagulation were taken two or three times a week throughout each study.

The diets fed during each of the three periods had the basic composition given in Table I. The various diets fed each patient were isocaloric. The weight of each patient was maintained constant throughout the four months of the entire study.

Table I.-Composition of Diet-Percentage of Total Calories

\begin{tabular}{|c|c|c|c|c|}
\hline & \multicolumn{3}{|c|}{ Diet } \\
\hline & & $\begin{array}{c}\text { Vegetable } \\
\text { Fat }\end{array}$ & $\begin{array}{l}\text { Egg Yolk, } \\
\text { Dairy Fat }\end{array}$ & $\begin{array}{l}\text { Low } \\
\text { Fat }\end{array}$ \\
\hline $\begin{array}{l}\text { Protein } \quad . \\
\text { Fat } \\
\text { Vegetable } \\
\text { Animal } \\
\text { Carbohydrate }\end{array}$ & $\begin{array}{l}. \\
\cdots \\
\cdots \\
\cdots\end{array}$ & $\begin{array}{l}14 \\
39 \\
21^{*} \\
18 \ddagger \\
47^{*}\end{array}$ & $\begin{array}{l}14 \\
37 \\
2 \\
35 \dagger \\
49\end{array}$ & $\begin{array}{l}12 \\
21 \\
7 \\
14 \ddagger \\
67\end{array}$ \\
\hline
\end{tabular}

* $95 \%$ of this fat was provided as corn oil, divided into three daily doses given with each meal.

$+95 \%$ of this fat was provided as egg yolk $(23 \%)$ and dairy fats $(72 \%)$.

₹ With the exception of $5 \mathrm{oz}$. (142 ml.) of skim milk daily, no dairy fats or eggs were used in this diet.

\section{Results}

Serum Lipid Values.-The highest mean cholesterol and mean phospholipid values were found during the period when the subjects received the diets rich in eggs and dairy fats (Table II). During the other two dietary

TABLE II.-Serum Cholesterol and Phospholipid Valuest

\begin{tabular}{|c|c|c|c|c|c|c|}
\hline \multirow{2}{*}{ Subject } & \multicolumn{3}{|c|}{$\begin{array}{c}\text { Cholesterol } \\
\text { (mg./100 ml.) }\end{array}$} & \multicolumn{3}{|c|}{$\begin{array}{l}\text { Phospholipid } \\
\text { (mg./100 ml.) }\end{array}$} \\
\hline & V.F.* & E.Y.* & L.F.* & V.F.* & E.Y.* & L.F.* \\
\hline $1\left\{\begin{array}{l}\text { Mean } \\
\text { No. of tests } \ldots\end{array}\right.$ & $\begin{array}{c}192 \\
(4)\end{array}$ & $\begin{array}{r}282 \\
(7)\end{array}$ & $\begin{array}{c}178 \\
(5)\end{array}$ & $\begin{array}{r}226 \\
(4)\end{array}$ & 261 & $\begin{array}{c}213 \\
(5)\end{array}$ \\
\hline $2\left\{\begin{array}{l}\text { Mean } \\
\text { No. of tests } \ldots\end{array}\right.$ & $\begin{array}{c}209 \\
(6)\end{array}$ & $\begin{array}{r}206 \\
(9)\end{array}$ & $\begin{array}{r}214 \\
(4)\end{array}$ & $\begin{array}{r}207 \\
(6)\end{array}$ & $\begin{array}{r}206 \\
(9)\end{array}$ & $\begin{array}{r}226 \\
\text { (4) }\end{array}$ \\
\hline $3\left\{\begin{array}{l}\text { Mean } \\
\text { No. of tests } \ldots\end{array}\right.$ & $\begin{array}{r}237 \\
(4)\end{array}$ & $\begin{array}{l}269 \\
(10)\end{array}$ & $\begin{array}{c}203 \\
(4)\end{array}$ & $\begin{array}{r}224 \\
(4)\end{array}$ & $\begin{array}{l}258 \\
(10)\end{array}$ & $\begin{array}{r}210 \\
(4)\end{array}$ \\
\hline $4\left\{\begin{array}{l}\text { Mean } \\
\text { No. of tests } \ldots\end{array}\right.$ & $\begin{array}{c}202 \\
(5)\end{array}$ & $\begin{array}{l}238 \\
(9)\end{array}$ & $\begin{array}{r}210 \\
(5)\end{array}$ & $\begin{array}{c}188 \\
(5)\end{array}$ & $\begin{array}{c}207 \\
(9)\end{array}$ & $\begin{array}{c}186 \\
(5)\end{array}$ \\
\hline $5\left\{\begin{array}{l}\text { Mean } \\
\text { No. of tests } \ldots\end{array}\right.$ & $\begin{array}{c}154 \\
(5)\end{array}$ & $\begin{array}{c}186 \\
(9)\end{array}$ & $\begin{array}{c}153 \\
(6)\end{array}$ & $\begin{array}{c}155 \\
(4)\end{array}$ & $\begin{array}{c}184 \\
(9)\end{array}$ & $\begin{array}{r}165 \\
(6)\end{array}$ \\
\hline $6\left\{\begin{array}{l}\text { Mean } \\
\text { No. of tests } \ldots\end{array}\right.$ & $\begin{array}{c}191 \\
(5)\end{array}$ & $\begin{array}{r}259 \\
(9)\end{array}$ & 210 & $\begin{array}{c}195 \\
(5)\end{array}$ & $\begin{array}{r}232 \\
(9)\end{array}$ & $\begin{array}{r}212 \\
\text { (1) }\end{array}$ \\
\hline $7\left\{\begin{array}{l}\text { Mean } \\
\text { No. of tests } \ldots\end{array}\right.$ & $\begin{array}{r}185 \\
(6) \\
\end{array}$ & $\begin{array}{r}235 \\
(7) \\
\end{array}$ & $\begin{array}{c}176 \\
(5)\end{array}$ & $\begin{array}{c}186 \\
(6)\end{array}$ & $\begin{array}{r}217 \\
(7) \\
\end{array}$ & $\begin{array}{r}191 \\
(5)\end{array}$ \\
\hline Mean of means .. & 196 & 239 & 192 & 197 & 224 & 201 \\
\hline $\begin{array}{l}\text { tests on paired } \\
\text { differences } \\
\text { between means: } \\
\text { E.Y. v. V.F. . } \\
\text { E.Y. v. L.F. - } \\
\text { V.F. v. L.F. . }\end{array}$ & \multicolumn{3}{|c|}{$\begin{array}{l}\text { t } 3.88, \mathrm{P}<0.01 \\
\text { t } 3.58 . \mathrm{P}<0.02 \\
\text { t } 0.57, \mathrm{P}<0.6\end{array}$} & \multicolumn{3}{|c|}{$\begin{array}{l}\text { t } 5.19, \mathrm{P}<0.005 \\
\text { t } 2.69, \mathrm{P}<0.05 \\
\text { t } 0.82, \mathrm{P}<0.5\end{array}$} \\
\hline
\end{tabular}

*V.F. $=$ Diet rich in vegetable fat. E.Y. $=$ Diet rich in dairy fat and eggs. L.F. = Low-fat diet.

+ Values determined during periods when platelet survival studies were done. 
periods the mean cholesterol and mean phospholipid values were similar.

In-vitro Coagulation Tests.-For all five tests studied the coagulant activity of the blood was greatest during the period the subjects received the egg and dairy fat diet (Table III). The results given are in each instance the mean values for the 10-day period of the platelet survival studies. Comparison of the low-fat and vegetable-fat periods gives much less clear results, only one of the differences reaching the $5 \%$ level of significance.

Platelet Survival and Turnover.-Platelet half-life was shortest and platelet turnover greatest during the period when the subjects received the diets rich in dairy fats and eggs (Table IV). Platelet half-life was significantly longer on the low-fat diet than on the diets rich in vegetable fat or dairy fats and eggs. Mean platelet turnover was least during the period when the subjects received the low-fat diet.

Relationship between Serum Lipids, Coagulation Tests, and Platelet Survival and Turnover.-Mean values were computed for the serum phospholipid and cholesterol values for each of the 21 dietary periods. There was an excellent correlation between these two fractions $(r=+0.86, P=0.001)$. This was in keeping with the findings of other investigators (J. A. Little, 1961, unpublished observations). For the other tests none of the values obtained is impressive (Table V). However, many of these tests have a high experimental error which may mask significant correlations. It may be noted. however, that the trends are absolutely regular; for all 20 readings the highest blood lipid values are associated with more active coagulation tests, shorter platelet survival, and increased platelet turnover.

Table V.-Correlations Between Serum Lipids and Coagulation

\begin{tabular}{|c|c|c|c|c|}
\hline \multicolumn{3}{|c|}{ Coagulation Test } & \multirow[b]{2}{*}{$\begin{array}{c}\text { Serum Cholesterol } \\
r=0.31 \\
r=0.12 \\
r=0.47 * \\
r+0.29 \\
r=0.15 \\
r+0.28 \\
r=0.40 \\
r=0.30 \\
r+0.53 * \\
r+0.37\end{array}$} & \multirow[b]{2}{*}{\begin{tabular}{|c|c|} 
Serum Phospholipid \\
$r=0.33$ \\
$r=0.29$ \\
$r=0.29$ \\
$r \quad+0.27$ \\
$r=0.05$ \\
$r+0.37$ \\
$r=0.17$ \\
$r=0.06$ \\
$r=0.40$ \\
$r+0.36$
\end{tabular}} \\
\hline $\begin{array}{l}\text { Clottir } \\
\text { Prothr } \\
\text { Plasm } \\
\text { Platele } \\
\text { ", } \\
\text { ", } \\
\text { ", } \\
\text { ", }\end{array}$ & 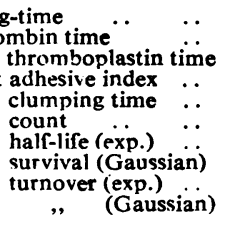 & $\begin{array}{l}. . \\
\because . \\
\therefore \\
\because . \\
\therefore \\
\cdots \\
\cdots\end{array}$ & & \\
\hline
\end{tabular}

\section{Discussion}

It has been recognized for many years that there is a relationship between diet, serum lipids, and atherosclerosis. It has been established that those foods which in man raise the serum lipids also accelerate the rate of development of atherosclerosis in a variety of experimental animals (Katz, Stamler, and Pick, 1958 ; Rowsell,

TABLE III.-Diet and In vitro Coagulation Tests*

\begin{tabular}{|c|c|c|c|c|c|c|c|c|c|c|c|c|c|c|c|}
\hline \multirow{2}{*}{ Subject } & \multicolumn{3}{|c|}{$\begin{array}{c}\text { Whole-blood Clotting- } \\
\text { time (Minutes) }\end{array}$} & \multicolumn{3}{|c|}{$\begin{array}{c}\text { One-stage Prothrombin } \\
\text { Time (Seconds) }\end{array}$} & \multicolumn{3}{|c|}{$\begin{array}{c}\text { Plasma Thromboplastin } \\
\text { Time (Seconds) }\end{array}$} & \multicolumn{3}{|c|}{$\begin{array}{l}\text { Platelet Adhesive } \\
\text { Index }\end{array}$} & \multicolumn{3}{|c|}{$\begin{array}{l}\text { Platelet Clumping } \\
\text { Time (Seconds) }\end{array}$} \\
\hline & V.F. & E.Y. & L.F. & V.F. & E.Y. & L.F. & V.F. & E.Y. & L.F. & V.F. & E.Y. & L.F. & V.F. & E.Y. & L.F. \\
\hline $1\left\{\begin{array}{l}\text { Mean } \\
\text { No. of tests }\end{array}\right.$ & $\begin{array}{l}12 \cdot 8 \\
(9)\end{array}$ & $\begin{array}{l}10 \cdot 2 \\
(9)\end{array}$ & $\begin{array}{l}12 \cdot 2 \\
(9)\end{array}$ & $\begin{array}{l}15 \cdot 0 \\
(4)\end{array}$ & $\begin{array}{l}14 \cdot 3 \\
(7)\end{array}$ & $\begin{array}{l}14 \cdot 4 \\
(5)\end{array}$ & $\begin{array}{l}11 \cdot 7 \\
(3)\end{array}$ & $\begin{array}{l}9 \cdot 0 \\
(5)\end{array}$ & $\begin{array}{l}11 \cdot 9 \\
(5)\end{array}$ & $\begin{array}{r}1 \cdot 22 \\
(3)\end{array}$ & $\begin{array}{r}1 \cdot 24 \\
(5)\end{array}$ & $\begin{array}{c}1 \cdot 12 \\
(5)\end{array}$ & $\begin{array}{l}216 \\
(10)\end{array}$ & $\begin{array}{l}212 \\
(8)\end{array}$ & $\begin{array}{r}228 \\
(9)\end{array}$ \\
\hline $2\left\{\begin{array}{l}\text { Mean } \\
\text { No. of tests }\end{array}\right.$ & $\begin{array}{l}12 \cdot 6 \\
(8)\end{array}$ & $\begin{array}{l}10 \cdot 7 \\
(7)\end{array}$ & $\begin{array}{l}14 \cdot 8 \\
(10)\end{array}$ & $\begin{array}{l}15 \cdot 1 \\
(5)\end{array}$ & $\begin{array}{l}14 \cdot 9 \\
(9)\end{array}$ & $\begin{array}{l}14 \cdot 9 \\
(9)\end{array}$ & $\begin{array}{l}10 \cdot 9 \\
(3)\end{array}$ & $\begin{array}{l}10 \cdot 7 \\
(5)\end{array}$ & $\begin{array}{l}11 \cdot 5 \\
(9)\end{array}$ & $\begin{array}{l}0.85 \\
(5)\end{array}$ & $\begin{array}{l}1.01 \\
(5)\end{array}$ & $\begin{array}{c}1 \cdot 10 \\
(7)\end{array}$ & $\begin{array}{r}222 \\
(7)\end{array}$ & $\begin{array}{l}233 \\
(8)\end{array}$ & $\begin{array}{r}522 \\
(10)\end{array}$ \\
\hline $3\left\{\begin{array}{l}\text { Mean } \\
\text { No. of tests }\end{array}\right.$ & $\begin{array}{l}13 \cdot 7 \\
(9)\end{array}$ & $\begin{array}{l}12 \cdot 6 \\
(8)\end{array}$ & $\begin{array}{l}17 \cdot 0 \\
(10)\end{array}$ & $\begin{array}{c}15 \cdot 2 \\
(4)\end{array}$ & $\begin{array}{l}14 \cdot 9 \\
(9)\end{array}$ & $\begin{array}{l}14 \cdot 8 \\
(10)\end{array}$ & $\begin{array}{l}10 \cdot 2 \\
(4)\end{array}$ & $\begin{array}{c}10 \cdot 2 \\
(6)\end{array}$ & $\begin{array}{l}11 \cdot 0 \\
(9)\end{array}$ & $\begin{array}{c}0.94 \\
(4)\end{array}$ & $\begin{array}{l}1 \cdot 37 \\
(5)\end{array}$ & $\begin{array}{c}0.99 \\
(8)\end{array}$ & $\begin{array}{l}262 \\
(8)\end{array}$ & $\begin{array}{l}265 \\
(9)\end{array}$ & $\begin{array}{l}465 \\
(10)\end{array}$ \\
\hline $4\left\{\begin{array}{l}\text { Mean } \\
\text { No. of tests }\end{array}\right.$ & $\begin{array}{l}14 \cdot 9 \\
(8)\end{array}$ & $\begin{array}{l}15 \cdot 1 \\
(9)\end{array}$ & $\begin{array}{l}13 \cdot 6 \\
(9)\end{array}$ & $\begin{array}{c}15 \cdot 2 \\
(4)\end{array}$ & $\begin{array}{l}15 \cdot 3 \\
(7)\end{array}$ & $\begin{array}{c}15 \cdot 1 \\
(5)\end{array}$ & $\begin{array}{l}14 \cdot 1 \\
(3)\end{array}$ & $\begin{array}{l}10 \cdot 1 \\
(5)\end{array}$ & $\begin{array}{c}11 \cdot 1 \\
(5)\end{array}$ & $\begin{array}{l}0.86 \\
(4)\end{array}$ & $\begin{array}{l}1 \cdot 34 \\
(6)\end{array}$ & $\begin{array}{c}1 \cdot 32 \\
(5)\end{array}$ & $\begin{array}{l}304 \\
(9)\end{array}$ & $\begin{array}{l}201 \\
(9)\end{array}$ & $\begin{array}{l}258 \\
(8)\end{array}$ \\
\hline $5\left\{\begin{array}{l}\text { Mean } \\
\text { No. of tests }\end{array}\right.$ & $\begin{array}{l}13 \cdot 2 \\
(9)\end{array}$ & $\begin{array}{l}14 \cdot 6 \\
(9)\end{array}$ & $\begin{array}{l}12 \cdot 9 \\
(8)\end{array}$ & $\begin{array}{l}15 \cdot 0 \\
(3)\end{array}$ & $\begin{array}{l}14 \cdot 6 \\
(7)\end{array}$ & $\begin{array}{l}14 \cdot 6 \\
(6)\end{array}$ & $\begin{array}{c}11 \cdot 4 \\
(3)\end{array}$ & $\begin{array}{l}9 \cdot 6 \\
(5)\end{array}$ & $\begin{array}{l}10 \cdot 4 \\
(5)\end{array}$ & $\begin{array}{l}1.00 \\
(5)\end{array}$ & $\begin{array}{l}1 \cdot 13 \\
(5)\end{array}$ & $\begin{array}{c}1.08 \\
(5)\end{array}$ & $\begin{array}{l}331 \\
(8)\end{array}$ & $\begin{array}{l}210 \\
(9)\end{array}$ & $\begin{array}{l}258 \\
(9)\end{array}$ \\
\hline $6\left\{\begin{array}{l}\text { Mean } \\
\text { No. of tests }\end{array}\right.$ & $\begin{array}{c}12 \cdot 8 \\
(8)\end{array}$ & $\begin{array}{c}11 \cdot 1 \\
(8)\end{array}$ & $\begin{array}{l}15 \cdot 1 \\
(10)\end{array}$ & $\begin{array}{c}15 \cdot 3 \\
(3)\end{array}$ & $\begin{array}{l}14.9 \\
(9)\end{array}$ & $\begin{array}{l}14 \cdot 8 \\
(10)\end{array}$ & $\begin{array}{l}10 \cdot 0 \\
(3)\end{array}$ & $\begin{array}{l}10 \cdot 2 \\
(5)\end{array}$ & $\begin{array}{l}12 \cdot 2 \\
(9)\end{array}$ & $\begin{array}{c}1 \cdot 02 \\
(4)\end{array}$ & $\begin{array}{l}0.93 \\
(5)\end{array}$ & $\begin{array}{c}1 \cdot 01 \\
(7)\end{array}$ & $\begin{array}{l}291 \\
(8)\end{array}$ & $\begin{array}{l}268 \\
(8)\end{array}$ & $\begin{array}{l}537 \\
(10)\end{array}$ \\
\hline $7\left\{\begin{array}{l}\text { Mean } \\
\text { No. of tests }\end{array}\right.$ & $\begin{array}{c}14 \cdot 0 \\
(8)\end{array}$ & $\begin{array}{c}12 \cdot 7 \\
(7)\end{array}$ & $\begin{array}{c}13 \cdot 1 \\
(9)\end{array}$ & $\begin{array}{c}15 \cdot 7 \\
(3)\end{array}$ & $\begin{array}{c}14.9 \\
(7)\end{array}$ & $\begin{array}{c}15 \cdot 3 \\
(5)\end{array}$ & $\begin{array}{l}12 \cdot 8 \\
(3)\end{array}$ & $\begin{array}{l}9 \cdot 7 \\
(5)\end{array}$ & $\begin{array}{c}10 \cdot 6 \\
(5)\end{array}$ & $\begin{array}{c}1 \cdot 18 \\
(5)\end{array}$ & $\begin{array}{l}1.26 \\
(6)\end{array}$ & $\begin{array}{l}1 \cdot 10 \\
(5)\end{array}$ & $\begin{array}{l}301 \\
(8)\end{array}$ & $\begin{array}{l}187 \\
(9)\end{array}$ & $\begin{array}{l}238 \\
(8)\end{array}$ \\
\hline $\begin{array}{c}\text { Appropriate } \\
\text { means of means }\end{array}$ & $13 \cdot 4$ & $12 \cdot 3$ & $14 \cdot 0$ & $15 \cdot 2$ & $14 \cdot 8$ & $14 \cdot 8$ & $11 \cdot 5$ & $9 \cdot 9$ & $11 \cdot 2$ & 1.01 & $1 \cdot 18$ & $1 \cdot 10$ & 272 & 223 & 335 \\
\hline $\begin{array}{l}\text { tests on paired } \\
\text { differences: } \\
\text { E.Y. v. V.F. } \\
\text { E.Y. v. L.F. } \\
\text { V.F. v. L.F. }\end{array}$ & \multicolumn{3}{|c|}{$\begin{array}{l}\text { t } 2.02, P<0.1 \\
\text { t } 1.77, P<0.2 \\
\text { t } 0.93, P<0.4\end{array}$} & \multicolumn{3}{|c|}{$\begin{array}{l}\text { t } 3.68, P<0.02 \\
\text { t } 0.000, P<0.001 \\
\text { t } 6.16, P<\end{array}$} & \multicolumn{3}{|c|}{$\begin{array}{l}\text { t } 2.72, P<0.05 \\
\text { t } 4.09, P<0.01 \\
\text { t } 0.37, P<0.8\end{array}$} & \multicolumn{3}{|c|}{$\begin{array}{l}\text { t } 2.18, P<0.1 \\
\text { t } 1.31, P<0.3 \\
\text { t } 1.23, P<0.3\end{array}$} & \multicolumn{3}{|c|}{$\begin{array}{l}\text { t } 2.21, P<0.1 \\
\text { t } 3.82, P<0.01 \\
\text { t } 1.18, P<0.3\end{array}$} \\
\hline
\end{tabular}

TABLE IV.-Diet and Platelet Survival and Turnover

\begin{tabular}{|c|c|c|c|c|c|c|c|c|c|c|c|c|c|c|c|}
\hline \multirow[t]{2}{*}{ Subject } & \multicolumn{3}{|c|}{$\begin{array}{c}\text { Mean* Platelet } \\
\text { Count } \\
\text { (Thousands/c.mm.) }\end{array}$} & \multicolumn{3}{|c|}{$\begin{array}{l}\text { Platelet Half-life } \\
\text { Exponential } \\
\text { (Days) }\end{array}$} & \multicolumn{3}{|c|}{$\begin{array}{c}\text { Platelet Turnover } \\
\text { Exponential } \\
\text { (Thousands'c.mm.'Day) }\end{array}$} & \multicolumn{3}{|c|}{$\begin{array}{c}\text { Platelet Survival } \\
\text { Gaussian } \\
\text { (Days) }\end{array}$} & \multicolumn{3}{|c|}{$\begin{array}{c}\text { Platelet Turnover } \\
\text { Gaussian } \\
\text { (Thousands/c.mm./Day) }\end{array}$} \\
\hline & V.F. & E.Y. & L.F. & V.F. & E.Y. & L.F. & V.F. & E.Y. & L.F. & V.F. & E.Y. & L.F. & V.F. & E.Y. & L.F. \\
\hline $\begin{array}{l}1 \\
2 \\
3 \\
4 \\
5 \\
6 \\
7\end{array}$ & $\begin{array}{l}250 \cdot 0 \\
192 \cdot 0 \\
260 \cdot 0 \\
147 \cdot 5 \\
150 \cdot 0 \\
225 \cdot 0 \\
248 \cdot 0\end{array}$ & $\begin{array}{l}250 \cdot 0 \\
200 \cdot 0 \\
234 \cdot 0 \\
188 \cdot 6 \\
178 \cdot 3 \\
182 \cdot 0 \\
355 \cdot 0\end{array}$ & $\begin{array}{l}204 \cdot 0 \\
210 \cdot 0 \\
215 \cdot 7 \\
218 \cdot 3 \\
184 \cdot 0 \\
192 \cdot 9 \\
286 \cdot 7\end{array}$ & $\begin{array}{l}4 \cdot 44 \\
3 \cdot 99 \\
4 \cdot 80 \\
2 \cdot 98 \\
4 \cdot 82 \\
3 \cdot 55 \\
3 \cdot 54\end{array}$ & $\begin{array}{l}4 \cdot 12 \\
2 \cdot 90 \\
2 \cdot 86 \\
2 \cdot 95 \\
2 \cdot 98 \\
2 \cdot 02 \\
3 \cdot 39\end{array}$ & $\begin{array}{l}4 \cdot 50 \\
4 \cdot 45 \\
5 \cdot 64 \\
3 \cdot 88 \\
5 \cdot 36 \\
5 \cdot 71 \\
3 \cdot 92\end{array}$ & $\begin{array}{l}39 \cdot 05 \\
33 \cdot 35 \\
37 \cdot 51 \\
34 \cdot 36 \\
21 \cdot 55 \\
43 \cdot 93 \\
48 \cdot 59\end{array}$ & $\begin{array}{l}47 \cdot 08 \\
47 \cdot 83 \\
56 \cdot 80 \\
44 \cdot 30 \\
41 \cdot 49 \\
62 \cdot 38 \\
72 \cdot 61\end{array}$ & $\begin{array}{l}31 \cdot 45 \\
32.69 \\
26 \cdot 52 \\
39 \cdot 05 \\
23.79 \\
23.42 \\
50 \cdot 71\end{array}$ & $\begin{array}{l}12 \cdot 50 \\
11 \cdot 31 \\
13 \cdot 42 \\
10 \cdot 46 \\
12 \cdot 60 \\
11 \cdot 19 \\
11 \cdot 15\end{array}$ & $\begin{aligned} 11 \cdot 65 \\
10 \cdot 46 \\
11 \cdot 34 \\
10 \cdot 81 \\
10 \cdot 85 \\
9.96 \\
11 \cdot 21\end{aligned}$ & $\begin{array}{l}12.24 \\
13.14 \\
13.56 \\
11 \cdot 52 \\
13.79 \\
13.88 \\
11.67\end{array}$ & $\begin{array}{l}20 \cdot 00 \\
16 \cdot 97 \\
19 \cdot 38 \\
14 \cdot 10 \\
11 \cdot 91 \\
20 \cdot 11 \\
22 \cdot 25\end{array}$ & $\begin{array}{l}21 \cdot 46 \\
19 \cdot 12 \\
20 \cdot 64 \\
17 \cdot 44 \\
16 \cdot 43 \\
18 \cdot 26 \\
31 \cdot 61\end{array}$ & $\begin{array}{l}16.66 \\
15.98 \\
15.90 \\
18.95 \\
13.34 \\
13.90 \\
24.57\end{array}$ \\
\hline Mean & $210 \cdot 4$ & $226 \cdot 8$ & 215.9 & 4.02 & 3.03 & $4 \cdot 78$ & 36.91 & $52 \cdot 50$ & $32 \cdot 52$ & $11 \cdot 80$ & 10.90 & $12 \cdot 83$ & $17 \cdot 82$ & $20 \cdot 72$ & $17 \cdot 04$ \\
\hline $\begin{array}{l}\text { t tests on } \\
\text { paired } \\
\text { differences : } \\
\text { E.Y. v. V.F. } \\
\text { E.Y. v. L.F. } \\
\text { V.F. v. L.F. }\end{array}$ & \multicolumn{3}{|c|}{$\begin{array}{l}\text { t } 0.88, \mathrm{P}<0.5 \\
\text { t } 0.82, \mathrm{P}<0.5 \\
\text { t } 0.32, \mathrm{P}<0.8\end{array}$} & \multicolumn{3}{|c|}{$\begin{array}{l}\text { t } 3.19, \mathrm{P}<0.02 \\
\text { t } 3.72, \mathrm{P}<0.01 \\
\text { t } 2.98, \mathrm{P}<0.025\end{array}$} & & $\begin{array}{l}31, \mathrm{P}< \\
57, \mathrm{P}< \\
28, \mathrm{P}<\end{array}$ & & \multicolumn{3}{|c|}{$\begin{array}{l}\text { t } 2.71, P<0.05 \\
\text { t } 3.76, P<0.01 \\
\text { t } 2.68, P<0.05\end{array}$} & \multicolumn{3}{|c|}{$\begin{array}{l}\text { t } 2.20, P<0.1 \\
\text { t } 3.67, P<0.02 \\
\text { t } 0.53, P<0.7\end{array}$} \\
\hline
\end{tabular}

* The mean of five or more readings. 
Downie, and Mustard, 1960). The evidence in man is mainly contingent, and Yudkin (1957) has stressed the dangers of a posteriori inferences of causality of this kind. Appreciation of the role of thrombi or encrustations in the development of atherosclerosis and its complications has led to studies of the effect of diet on blood coagulation. Most investigations have shown that dietary fat influences blood-clotting in vitro. There have been a few contrary reports. Two of five such reports, however, contain evidence overlooked by their authors indicating that alimentary lipaemia after meals rich in dairy fat may in fact accelerate clotting as measured by in vitro tests (Mustard, 1961). It is perhaps dangerous to infer what effects dietary fat has on autochthonous coagulation from the study of in vitro phenomena. It is because of this that we have studied platelet survival and turnover as measures of the in vivo activity of clotting, in particular of the early stages. Further, we have studied the effect of diet over a number of weeks rather than the acute effects of a meal rich in fat.

These findings show that blood coagulation as judged by platelet survival and turnover is more active when subjects received a diet rich in dairy fats and eggs than with other diets used; parallel changes were found with the in vitro tests. Mayer and Connell (1958) and Buzina, Karvonen, Roine, and Turpeinen (1961) have reported that the replacement of saturated fat in the diet by unsaturated fat leads to a lengthening of the wholeblood clotting-time in silicone-coated tubes. The results for the whole-blood clotting-time in the present study showed a similar trend. There was no clear-cut difference between the effects of the low-fat and vegetablefat diets, but it is of some interest that mean platelet survival was significantly longer when the subjects received the former. A low-fat diet has been reported to be associated with decreased platelet adhesiveness (McDonald and Edgill, 1958). We have found similar, but inconclusive, results in this small series. It is of interest that the association of diminished platelet adhesiveness with prolonged survival has been previously observed in another connexion (Murphy and Mustard, 1962).

The factors in the diet responsible for the differences found in the present study are not clearly defined. The difference between the vegetable-fat and dairy-fat diets seems to lie in the lipid content. It is known that dairy fats and eggs contain coagulant-active phospholipid not present in corn oil. Whether it is the difference in phospholipid, fatty acid, or other aspects of fat composition which account for this can be determined only by further study. It is not impossible that the effects of the low-fat diet may be in part attributable to the increase in carbohydrate intake necessary to keep the diets isocaloric.

We previously reported that there was an increase in what could be considered factor IX activity during the period of lipaemia following a meal rich in dairy fat and eggs (Mustard, 1957). In the present study there were differences between the mean values for the plasma thromboplastin time in the various dietary periods. We have previously found that this test reflects changes in factor IX activity (Mustard, 1958). Such changes occur early in blood coagulation and are associated with changes in platelet adhesiveness (Murphy and Mustard, 1962). Some observers have reported that activation of this factor is influenced by phospholipids (Seegers and
Johnson, 1956 ; Surgenor, Steele, and Wallach, 1959). This suggests one mechanism by which the effect of diet could be mediated.

The relationship between the actual serum lipid levels and the various clotting-tests is unimpressive. Lack of better relationship may be due to the experimental error of the tests used or to the fact that those lipid components with coagulant activity were not measured separately. It is doubtful if these changes in clotting are sufficient by themselves to lead to the formation of occlusive thrombi. In experimental animals parenteral administration of dilute thrombin or tissue thromboplastin can lead to marked acceleration of the clotting mechanism, even to the point of exhaustion of clotting factors such as factor VIII, but without producing evidence of occlusive thrombosis (Brayton and Zucker, 1957 ; Penick, Roberts, Webster, and Brinkhous, 1958). However, it is possible that in subjects with marked vessel-wall disease the coagulation changes might facilitate the formation of occlusive thrombi, particularly if there are also local disturbances of blood-flow.

The relationship of the changes found in this study to atherogenesis is more speculative. We have suggested elsewhere that the encrustation hypothesis be modified to include the formation of platelet microthrombi (Mustard, Downie, Murphy, and Rowsell, 1961). If the platelet normally tends to adhere to the vascular endothelium increased platelet adhesiveness would be reflected in increased platelet turnover and shortened platelet survival. The only evidence on this point at the moment is indirect. Fatty acids have been found to increase thrombus formation in in vitro experiments (Connor and Poole, 1961).

Whatever the nature of this relationship, it is important to emphasize that dietary factors which have been long known to have a hypercholesterolaemic effect also accelerate blood coagulation. Although we do not yet know the effect of dietary management on the development of atherosclerosis and its complications in man, we would like to point out that the changes in platelet economy produced in these subjects by the various diets were as great as or greater than those we have been able to achieve with intensive dicoumarol therapy (Murphy and Mustard, 1961).

During the last war it was found that although the people in Norway, Sweden, Finland, and Denmark had a significant reduction in their intake of fat, Denmark showed no reduction in mortality from complications of vascular disease; Denmark was the one country in which there was not a significant reduction in the consumption of dairy fats and eggs (Malmros, 1950). A later study from Denmark (Geill, Hansen, and Lund, 1960) has reported that a group of patients on a diet rich in vegetable fat showed fewer episodes of thrombosis than a comparable control group left on a diet rich in animal fats. Briggs, Rubenberg, O'Neal, Thomas, and Hartroft (1960) have reported that patients with peptic ulcers maintained on diets rich in dairy fats show an increased incidence of myocardial infarction. These findings would suggest that dietary management might be a more effective long-term treatment for patients with atherosclerotic vessel disease and its complications than anticoagulants.

\section{Summary}

Dietary studies of some weeks' duration were performed on seven male subjects. In vitro tests of 
blood coagulation were most active and platelet survival shortest when the diet was rich in dairy fats and eggs. When the diet contained little fat converse results were obtained. A diet rich in vegetable fat produced intermediate results. The serum cholesterol and phospholipid values were highest with the dairy fat and egg yolk diet. However, poor correlations were found between the level of the blood lipids and the coagulation tests. Thus, extended studies have shown that dietary control influences not only serum lipid values but also blood coagulation in vitro and platelet economy in vivo.

We are indebted to Mr. M. Boroscki, Mr. M. Kovacs, Mrs. Sheila Newton, Mrs. Jane Schulze, and Mrs. Norma Ruth for their patience and industry. We gratefully acknowledge the advice and assistance of Miss R. Robidoux and Miss D. E. McNaughton in designing and preparing the diets used in this study. The Department of Veterans Affairs and Armour Pharmaceutical Company provided grants which aided this study.

\section{REFERENCES}

Brayton, R. G., and Zucker, M. B. (1957). Proc. Soc. exp. Biol. (N,Y), 96, 418

Briggs, R. D., Rubenberg, M. L., O'Neal, R. M., Thomas, W. A. and Hartroft, W. S. (1960).' Circulation, 21, 538.

Buzina, R., Karvonen, M. J., Roine, P., and Turpeinen, O. (1961). Lancet, 2, 287.

Connor, W. E., and Poole, J. C. F. (1961). Quart. J. exp. Physiol., 46, 1.

Geill, T., Hansen, P. F., and Lund, E. (1960). Nature (Lond.), $185,330$.

Katz, L. N., Stamler, J., and Pick, R. (1958). Nutrition and Atherosclerosis. Lea and Febiger, Philadelphia.

Leeksma, C. H. W., and Cohen, J. A. (1956). J. clin. Invest., $35,964$.

McDonald, L., and Edgill, M. (1958). Lancet, 1, 996.

Malmros, H. (1950). Acta med. scand., Suppl. 246, p. 137.

Mayer, G., and Connell, W. F. (1958). Circulation, 18, 496.

Murphy, E. A., and Mustard, J. F. (1960). Circulat. Res., 8 1187.

— (1961). Ibid., 9, 402.

(1962). Circulation, 25, 114

Mustard, J. F. (1956). Brit. J. Haemat., 2, 17.

(1957). Canad. med. Ass. J., 77, 308.

(1958). Ibid., 79, 554

(1961). Ibid., 85, 621.

Downie, H. G., Murphy, E. A., and Rowsell, H. C. (1961) In Blood Platelets, edited by S. Johnson, p. 191. Little, Brown, Boston.

O'Brien, J. R. (1957). Amer. J. med. Sci., 234, 373. (1958). Progr. cardiovas. Dis., 1, 151.

Penick, G. D., Roberts, H. R., Webster, W. P., and Brinkhous, K. M. (1958). Arch. Path., 66, 708.

Rowsell, H. C., Downie, H. G., and Mustard, J. F. (1960). Canad. med. Ass. J., 83, 1175 .

Seegers, W. H., and Johnson, S. A. (1956). Amer. J. Physiol., 184, 259.

Sperry, W. M., and Webb, M. (1950). J. biol. Chem., 187, 97. Surgenor, D. M., Steele, B. B., and Wallach, D. F. H. (1959). Thrombos, Diathes. Haemorrh. (Stuttg.), 3, 215.

Thomas, C. B., and Eisenberg, F. (1957). J. chron. Dis., 6, 1. Yudkin, J. (1957). Lancet, 2, 155.

Zilversmit, D. B., and Davis, A. K. (1950). J. Lab. clin. Med. 35,155 .

Islington Public Libraries have issued a book list, Family Wise, which provides a broad selection of books on marriage, family planning, pregnancy and childbirth, parenthood, child care, sex education, and related topics in stock in these libraries. The book list is available free of charge from any Islington library. A copy will be sent to anyone who cares to write for it, enclosing $2 \mathrm{~d}$. postage, to the Islington Central Library, 68 Holloway Road, London N.7.

\section{VEGANISM : A CLINICAL SURVEY WITH OBSERVATIONS ON VITAMIN-B 12 METABOLISM}

BY

\author{
A. D. M. SMITH, M.B., B.Sc., M.R.C.P.*
}

Leverhulme Research Scholar, Institute of Clinical Research, Middlesex Hospital Medical School and Central Middlesex Hospital, London

A vegan can be defined as an extreme vegetarian who excludes all animal protein from his diet. Dairy produce is acceptable to the ordinary lactovegetarian but not to the vegan, who may in certain cases feel that any animal contact is to be abhorred for ethical reasons and replace all articles of clothing and household equipment possessing an animal origin. The vast majority of these subjects neither smoke nor drink, and this renders them eminently suitable for clinical investigation, bearing in mind the possibility of vitamin- $B_{12}$ deficiency. Some previous observations from this viewpoint have already been published (Donath et al., 1953 ; Hardinge and Stare, 1954 ; Wokes et al., 1955). However, this previous work has been mainly preoccupied with the possible effects from various dietary deficiencies, and in the present paper it is hoped to offer a solution to the reverse of this context. The question seems to be, not why do some people on this form of diet develop vitamin- $B_{12}$ deficiency, but why many subjects do not. In an attempt to obtain an answer a small group of vegans were asked to co-operate in a clinical survey in the hope that some explanation of this apparent lack of deficiency might be forthcoming.

\section{Material and Methods}

Composition of Group.-Using a $100 \%$ criterion for veganism 12 subjects were found who agreed to the scheme of investigation proposed. They had lived on their rigorous diet for from 4 to 30 years, and their serum vitamin- $B_{13}$ levels, estimated by the Lactobacillus leichmannii method with cyanide extraction, ranged from 48 to $220 \mu \mu \mathrm{g} . / \mathrm{ml}$. and averaged $128 \pm 15 \mu \mu \mathrm{g} . / \mathrm{ml}$. (normal range from conventional viewpoint, 140-900 $\mu \mu \mathrm{g} . / \mathrm{ml}$.). This level compares closely with that obtained in the previous British series (Wokes et al., 1955) and has been reported previously (Wokes and Smith, 1961). Apart from routine serum vitamin-B $B_{12}$ assays the subjects were further analysed as follows.

History and Clinical Examination.-A detailed history was obtained from each volunteer. Particular attention was paid to symptoms that might have arisen from vitamin- $B_{12}$ deficiency, and other symptoms that were reported in the previous British series were also carefully inquired into. A full clinical examination, which included an entire neurological survey, was then made.

Ancillary Investigations.-Full blood count, serum iron, serum proteins, and electroencephalographic examination were performed on all subjects with two exceptions (see Table). In addition those with cardiac murmurs were further screened by chest $x$-ray and electrocardiographic examinations.

\section{Results}

In this small group the notable feature of the histories obtained lay in the paucity of the symptoms. Only *Now at Westminster Hospital, London. 